\title{
Asymptomatic Fascioliasis
}

\author{
Seiji ADACHI, Kazuhiko Kotani*, Tetsu ShIMIZU**, Kiwamu TANAKA***, \\ Tatsunori SHIMIZU*** and Katsuo OKADA***
}

\begin{abstract}
A 72-year-old Japanese man displayed asymptomatic eosinophilia for 4 months. Computed tomography showed multiple space-occupying lesions in the liver. Zoonotic liver flukes were suspected based on occupational exposure to cattle, serological and radiological findings. Immunological examination was helpful in diagnosing the disease and laparoscopy was crucial in confirming Fasciola hepatica and excluding the possibility of malignant hepatic tumors. Human fascioliasis was finally diagnosed and praziquantel administered. Blood eosinophilia resolved within $\mathbf{4}$ months and liver tumors almost disappeared within 12 months. From our experience, laparoscopy with liver biopsy is very important for diagnosing human fascioliasis, particularly for asymptomatic fascioliasis.
\end{abstract}

(Internal Medicine 44: 1013-1015, 2005)

Key words: human fascioliasis, eosinophilia, laparoscopy, liver

\section{Introduction}

Infections with Fasciola Hepatica are worldwide zoonoses. Fasciola Hepatica can infect a wide variety of mammalian hosts, particularly sheep, goats and cattle. Human cases have been reported in South America, Europe, Africa, Australia, and the Far East. Recent estimates indicate a worldwide prevalence of 17 million cases (1), but human fascioliasis is rare in Japan. More than 100 cases had been reported until $1992(2)$.

\section{Case Report}

In October, 2003, a previously healthy 72-year-old Japanese man presented to a clinic with common cold. Blood tests revealed an increased eosinophil count. He was followed without medication because of a lack of symptoms, but eosinophilia was unchanged for 4 months. The clinic doctor then referred the patient to us for further evaluation of eosinophilia in February 2004. Blood tests showed leucocytosis $\left(11.6 \times 10^{9} / l\right)$ with $46.5 \%$ eosinophils, IgE levels of $9,620 \mathrm{IU} / \mathrm{ml}$ (normal, $\leqq 250 \mathrm{IU} / \mathrm{ml}$ ) and normal C-reactive protein levels. Liver function tests and tumor markers levels were all within reference ranges. Computed tomography showed multiple space-occupying lesions in the liver (Fig. 1). The biggest tumor formed a cluster. Multiple small spaceoccupying lesions were recognized under liver surface. These findings were suggestive of malignant metastases. Gastroduodenoscopy, colonoscopy and magnetic resonance cholangiopancreatography revealed no abnormalities.

The patient had often eaten a dropwort in his backyard. He worked on a farm and kept cows, so zoonotic liver flukes were suspected based on his lifestyle history, serological and radiological findings. However, the presence of eggs in stool samples and biliary tract drainage was not detected. Given radiological findings, malignant hepatic metastases of unknown origin and primary liver cancer (non-HBV, non-HCV type) were also considered. Laparoscopy revealed multiple yellowish-white nodules on the liver surface (Fig. 2). Demarcated eosinophilic abscess typical of human fascioliasis were observed on liver biopsy (Fig. 3). Immunological testing for human fascioliasis and other parasites using enzyme-linked immunosorbent assay revealed positive antiFasciola hepatica antibodies in serum. Human fascioliasis was diagnosed and praziquantel $60 \mathrm{mg} / \mathrm{kg} /$ day was administered for 5 days. Blood eosinophilia resolved within 4 months (about 5\% eosinophils). Liver tumors almost disappeared within 12 months, but positive anti-Fasciola hepatica

From the Department of General Internal Medicine, Tottori Central Prefectural Hospital, Tottori, *the Division of Health Administration and Promotion, Faculty of Medicine, Tottori University, Yonago, **the Department of Surgery, and ***the Department of Internal Medicine, Tottori Central Prefectural Hospital, Tottori

Received for publication January 19, 2005; Accepted for publication May 11, 2005

Reprint requests should be addressed to Dr. Seiji Adachi, the Department of General Internal Medicine, Tottori Central Prefectural Hospital, 730 Ezu, Tottori 680-0901 


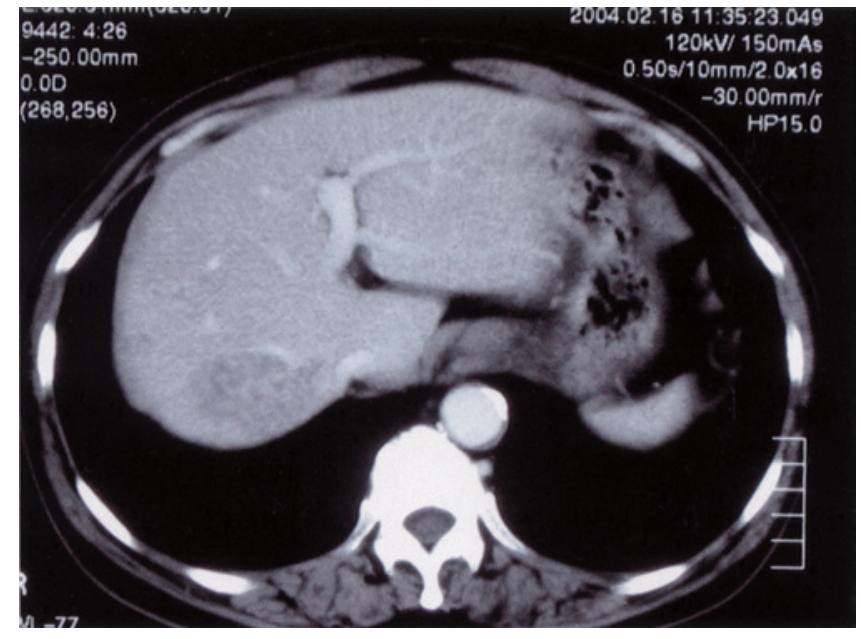

Figure 1. Computed tomography showing multiple spaceoccupying lesions in the liver.

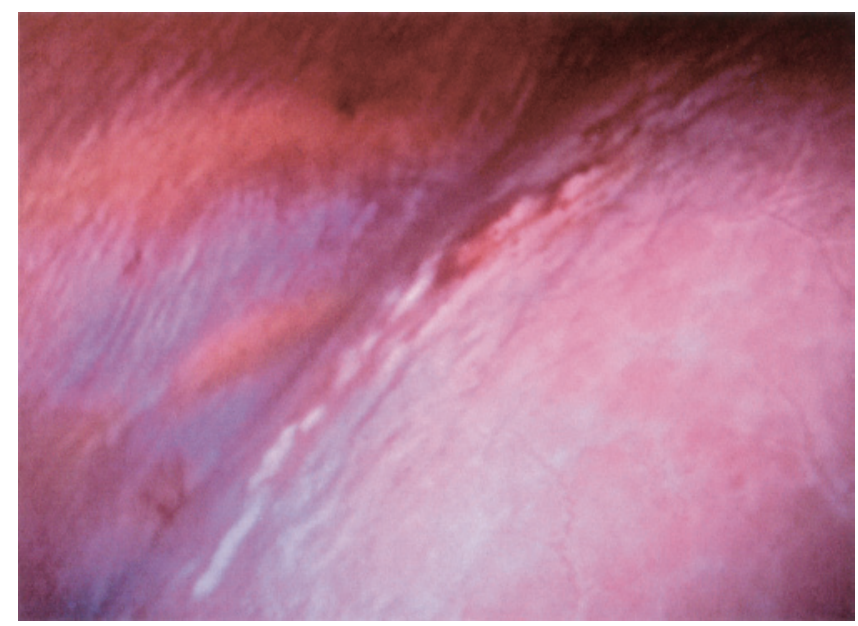

Figure 2. Laparoscopy showing multiple yellowish-white nodules on the liver surface.

antibodies in the serum still remained after the eradication. IgE levels were decreased to $1,570 \mathrm{IU} / \mathrm{ml}$, but were still high level after 12 months.

\section{Discussion}

Although typical symptoms of fascioliasis are upper abdominal pain and malaise (3), 15\% of patients are asymptomatic, as in the present case (4). Asymptomatic fascioliasis must therefore be considered when asymptomatic eosinophilia is encountered. Diagnosis and treatment are not easy for physicians with little experience of this disease, particularly if asymptomatic. Diagnosis has traditionally relied on the detection of the presence of eggs in fecal samples, but

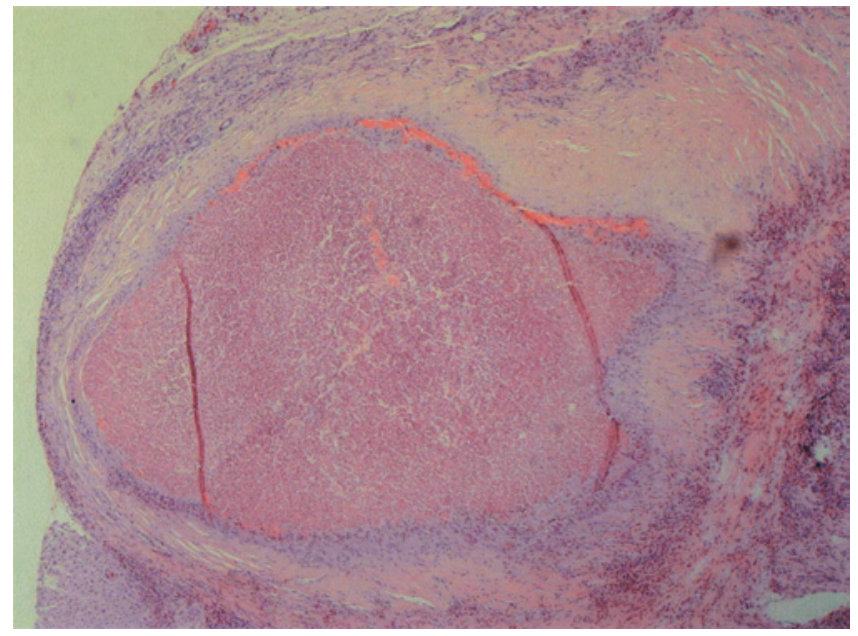

Figure 3. Demarcated eosinophilic abscess in liver tissue (HE stain, $\times 4$ ).

this method is often unreliable. Detection of serum antiflukeantibodies is popular (5), but positive results may only indicate past infection, particularly with asymptomatic fascioliasis.

If fascioliasis is suspected, past history is important, and radiological examination, sonography and magnetic resonance imaging could be performed for detection of abnormal lesions. Serum immunological examination will be helpful in diagnosing the disease, although again, positive results may simply indicate past infection. If hepatic tumors are detected, malignancy may be incompletely excluded from differential diagnoses. Laparoscopy can thus be crucial in diagnosing the presence of Fasciola hepatica, which is associated with multiple yellowish-white nodules $1-4 \mathrm{~mm}$ in diameter on the liver surface $(6,7)$. Pathological findings of demarcated eosinophilic abscess indicate the necrotic tunnel shaped areas created by the parasite as it penetrates the hepatic tissue. From our experience, laparoscopy with liver biopsy is very important for diagnosing human fascioliasis, particularly for asymptomatic fascioliasis.

\section{References}

1) Kasper DL, Braunwald E, Fauci AS, et al. Liver (Biliary) Flukes. In: Harrison's Online (Featuring the complete contents of Harrison's Principles of Internal Medicine. 16th Ed), Part 6, Section 19, Chapter 203. 2005 .

2) Ishii $Y$, Nakamura-Uchiyama F, Nawa Y. A praziquantel-ineffective fascioliasis case successfully treated with triclabendazole. Parasitol International 51: 205-209, 2002.

3) Saba R, Korkmanz M, Inan D, et al. Human fascioliasis. Clin Microbiol Infect 10: 385-387, 2004.

4) Haseeb AN, El-Shazly AM, Arafa MA, Morsy AT. Clinical laboratory and ultrasonography features of proven human fascioliasis. J Egypt Soc Parasitol 33: 397-412, 2003.

5) Carnevale S, Rodriguez MI, Santillan G, et al. Immunodiagnosis of human fascioliasis by an enzyme-linked immunosorbent assay (ELISA) 


\section{Asymptomatic Fascioliasis}

and a micro-ELISA. Clin Diagn Lab Immunol 8: 174-177, 2001.

6) Moreto M, Barron J. The laparoscopic diagnosis of the liver fascioliasis. Gastrointest Endosc 26: 147-149, 1980.
7) Uribarrena R, Borda F, Munoz M, Rivero-Puente A. Laparoscopic findings in eight cases of liver fascioliasis. Endoscopy 17: 137-138, 1985. 